\title{
Renal Allograft Survival in Transplant Recipients with Focal Segmental Glomerulosclerosis
}

\author{
Diane M. Cibrik', Bruce Kaplan², \\ Darrell A. Campbell ${ }^{2}$ and \\ Herwig-Ulf Meier Kriesche ${ }^{3, *}$ \\ Department of ${ }^{1}$ Medicine and ${ }^{2}$ Surgery, University of \\ Michigan, Ann Arbor, MI, USA, ${ }^{3}$ Department of Medicine, \\ Division of Nephrology, University of Florida, Gainsville, FL, \\ USA \\ ${ }^{*}$ Corresponding author: Herwig-Ulf Meier Kriesche, MD, \\ meierhu@medicine.ufl.edu
}

Previous literature suggests that the recurrence of focal segmental glomerulosclerosis (FSGS) after renal transplantation is more common in recipients who have received an HLA-identical living-related (LRD) transplant. To address the question if FSGS patients can safely receive a 6-antigen match LRD kidney transplant, we analyzed death-censored renal allograft survival data of FSGS patients from the United States Renal Data System database (USRDS).

Using the USRDS and the U.S. Scientific Renal Transplant Registry between the years 1988-97, we found 19259 adult primary renal transplant recipients, of which 2414 patients had FSGS as their primary diagnosis as compared to 16845 patients who had other types of glomerulonephritis (GN). A Cox proportional hazard model was used to estimate death-censored graft survival among FSGS patients with a zero mismatch LRD kidney transplant. The model included a triple interaction term comparing FSGS vs. GN vs. living donation (LD) vs. cadaveric donation (CAD) vs. zero mismatch (six antigen or HLA-identical) vs. mismatch. Annually adjusted death censored graft loss rates per 1000 patients (ADGL) were calculated.

Focal segmental glomerulosclerosis patients receiving a zero mismatch LRD kidney transplant had the lowest ADGL rate, losing 10.5 grafts per 1000 patients per year. Not significantly different but higher (14.3) was the ADGL rate for LD, zero mismatch GN recipients. The ADGL rate was significantly higher in FSGS recipients who received a LD, mismatched transplant (36.5). Focal segmental glomerulosclerosis patients who received a CAD, zero mismatched graft (44.1), or CAD, mismatched graft (63.2), had significantly higher ADGL rates.

Zero mismatch LRD kidney transplants are not a risk factor for graft loss in FSGS patients but are associated with significantly better death-censored graft survival as compared to CAD 6-antigen match or mismatched donations.

Key words: 6-antigen match, FSGS, graft survival, living donor, renal transplantation, zero mismatch
Received 3 April 2002, revised and accepted for publication 2 August 2002

\section{Introduction}

Focal segmental glomerulosclerosis (FSGS) is a common cause of nephrotic syndrome in the United States (1). The pathogenesis of FSGS remains unclear but has been attributed to a 'circulating factor' present in the serum of patients with FSGS (2). Recurrence of FSGS after transplantation is not unusual and is most likely to occur in those recipients who have progressed rapidly to end-stage renal disease (ESRD) from the time of diagnosis (3). Post-transplantation recurrence of FSGS in the kidney allograft can occur in up to $50 \%$ of renal allografts (4). In addition, allograft failure from recurrent FSGS is significant with the relative risk for graft loss of 2.25 and the 5 -year kidney survival rate of $34.2 \%$ for transplant recipients with recurrent FSGS (5). Attempts have been made to remove the 'FSGS factor' with plasmapheresis before renal transplantation or peri-operatively with variable success (6). Because of the substantial risk of renal allograft loss from recurrence of FSGS, it has been recommended that live donation be avoided (7).

Historically and today, FSGS patients have received kidney transplants from living-related donors (LRD). Surveying 89 U.S. transplant centers in the late 1970s, Zimmerman reported an $82 \%$ incidence of recurrent FSGS in transplant recipients who received at least a 4-antigen-matched LRD transplant, and concluded that HLA-identical sibling transplants should be avoided in this population (8). Subsequently, Morzycka et al. suggested that recurrent FSGS was more common in those renal transplant recipients who received LRD transplants (9). Current reference literature on renal transplantation continues to suggest that LRD transplants for patients with FSGS have a high rate of recurrence and hence, portend a worse outcome with such transplants as opposed to cadaveric transplants $(10,11)$. Several groups have examined graft loss from recurrent FSGS with conflicting results $(12,13)$. The above-mentioned studies suggest that recurrence of FSGS is more common in LRD and zero mismatch kidney transplants as compared to cadaveric and unmatched kidney transplants. As mentioned earlier, as recurrence of FSGS has been related to worse graft survival, these data suggest that a zero mismatch LRD kidney transplant should be avoided in patients with ESRD secondary to FSGS. In fact, this recommendation is widely held by U.S. transplant centers. 
Table 1: Cox model for death-censored graft loss

\begin{tabular}{llclll}
\hline Disease & Donor & Number of patients & Match & RR & $95 \% \mathrm{Cl}$ \\
\hline GN & LD & 979 & Full & ref. & - \\
GN & CAD & 436 & Full & 2.01 & $1.51-2.68$ \\
GN & LD & 4017 & Mis & 2.06 & $1.65-2.57$ \\
GN & CAD & 11413 & Mis & 3.77 & $3.03-4.69$ \\
FSGS & LD & 136 & Full & 0.78 & $0.39-1.54$ \\
FSGS & CAD & 72 & Full & 1.88 & $1.09-3.26$ \\
FSGS & LD & 624 & Mis & 2.53 & $1.89-3.39$ \\
FSGS & CAD & 1582 & Mis & 4.47 & $3.53-5.66$ \\
\hline
\end{tabular}

$\mathrm{GN}=$ glomerulonephritis, FSGS = focal segmental glomerulosclerosis, $\mathrm{LD}=$ living donor, $\mathrm{CAD}=$ cadaveric donation.

On the other hand, it has been documented previously in the literature that graft survival among LRD transplant recipients is superior to the graft survival of cadaveric (CAD) transplants, and that well-matched renal allografts have superior graft survival as compared to mismatched grafts (14). To determine whether the potentially higher risk for recurrence of FSGS outweighs the benefits of receiving a living related and/or well-matched kidney transplant, we examined the death-censored graft survival rates among FSGS patients held in the United States Renal Data System (USRDS) database. Thus, we used death-censored graft survival as the final study endpoint and not recurrence of FSGS.

\section{Methods}

To evaluate renal allograft survival in FSGS patients who received a zero mismatch LRD transplant, we analyzed 19259 adult primary renal transplant recipients registered in the USRDS database between 1988 and 1997. Of these patients, 2414 recipients had FSGS as their primary diagnosis as compared to 16845 patients with glomerulonephritis (GN). Glomerulonephritis patients were used as the reference population, as these patients were felt most likely to reflect a homogeneous population of patients without preexisting comorbidities, similar to those patients with FSGS.

A Cox proportional hazard model was used to estimate the adjusted deathcensored graft survival among FSGS and GN patients who received a zero mismatch LRD transplant. Estimates of graft survival among FSGS and GN patients were also obtained for HLA-mismatched LRD transplants, zero mismatch CAD transplants, and HLA-mismatched CAD transplants. A triple interaction term was included in the Cox model to look at the potential for effect-modification between FSGS vs. GN vs. living donation vs. CAD vs. zero mismatch (HLA-identical or 6-antigen match) vs. HLA-mismatch. In addition, the Cox model was adjusted for 11 covariates relevant to death-censored graft survival including donor and recipient age, recipient gender and race, panel reactive antibody (PRA), cold ischemic time (CIT), year of transplantation, time on dialysis, induction, calcineurin inhibition (FK506 vs. cyclosporine), and antiproliferative agents (azathioprine vs. mycophenolate mofetil). The primary study endpoint was adjusted death-censored graft survival. Patients were studied until graft loss, death, or loss to follow up, in which time they were censored. A probability of type 1 error $(\alpha)=0.05$ was considered to be the threshold of statistical significance. All statistical analyses were performed using SPSS software (version 10.0 for Windows 95; SPSS Inc., Chicago, IL).

\section{Results}

Table 1 displays the interaction terms for the Cox proportional hazard model for death-censored graft survival. Causes of ESRD that received a living donated (LD) kidney transplant are depicted in bold lettering as compared to those that received a CAD kidney transplant. In this model, GN patients who received a zero mismatch LD kidney transplant were considered as the reference group. Focal segmental glomerulosclerosis patients who received a zero mismatch LD kidney transplant had equivalent death-censored graft survival as compared to GN patients with a zero mismatch LD transplant (p-value not significant). In contrast to the GN patients with a zero mismatch LD transplant, GN and FSGS recipients who received a zero mismatch $C A D$ renal transplant had significantly worse death-censored graft survival. Focal segmental glomerulosclerosis and GN patients who received a mismatched LD renal transplant had significantly worse deathcensored graft survival, as did those FSGS and GN patients who received a mismatched CAD renal transplant. The relative risk of graft failure for FSGS patients who received a mismatched cadaveric renal transplant was more than three-fold higher than the GN reference group. Compared with FSGS patients receiving a zero mismatch LD renal transplant, FSGS patients who received a mismatched cadaveric kidney transplant had dramatically worse death-censored graft survival.

Figure 1 shows the differences in death-censored graft survival among FSGS and GN patients who received either a zero mismatched or a mismatched CAD or LD renal transplant. As shown in Figure 2, death-censored graft survival is similar among GN and FSGS patients who received a zero mismatch LD kidney transplant. Conversely, GN and FSGS patients who received a mismatched CAD kidney transplant had poor five-year graft survival. Thus, FSGS patients who received a zero mismatch LD kidney transplant had 15\% better graft survival than those FSGS patients who received a zero mismatch CAD kidney transplant. Additionally, deathcensored graft survival was $22 \%$ better in the FSGS recipients who received a zero mismatch LD renal transplant as compared to those FSGS recipients who received a mismatched $C A D$ renal transplant. 


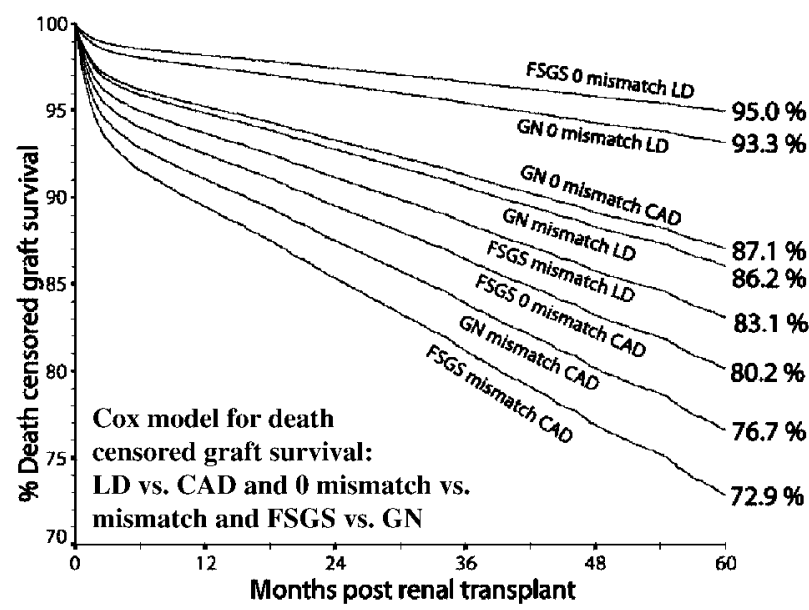

Figure 1: Five-year death-censored renal allograft survival in renal transplant recipients with either focal segmental glomerulosclerosis (FSGS) or glomerulonephritis and with either a zero mismatch or a HLA-mismatch vs. cadaveric donation or living donor (LD) kidney transplant

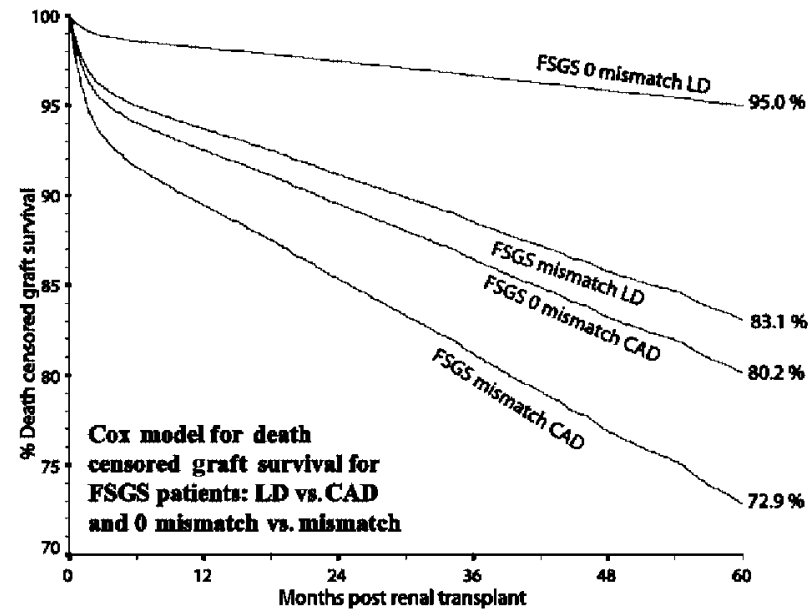

Figure 2: Five-year death-censored renal allograft survival in renal transplant recipients with focal segmental glomerulosclerosis (FSGS) and with either a zero mismatch, living donor (LD) kidney transplant; an HLA-mismatch, LD kidney transplant; a zero mismatch, cadaveric (CAD) kidney transplant; or an HLA-mismatch, CAD kidney transplant. GN= glomerulonephritis

Figure 2 shows the death-censored graft survival among FSGS patients who received either a zero mismatched or a mismatched LD renal transplant as compared to those FSGS patients who received either a zero mismatched or a mismatched CAD renal transplant. Focal segmental glomerulosclerosis recipients who received a zero mismatch LD kidney transplant had significantly superior death-censored graft survival (95\%) as compared to those FSGS recipients who received either a mismatch LD renal transplant (83.1\%) or either a zero mismatched or a mismatched CAD renal transplant (80.2\% vs. $72.9 \%$, respectively).
Table 2: Annually adjusted graft loss rates (per 1000 patient-years)

\begin{tabular}{llll}
\hline FSGS & LD & OMM & Rates \\
\hline Yes & yes & yes & 10.5 \\
No & yes & yes & 14.3 \\
No & no & yes & 27.9 \\
No & yes & no & 29.7 \\
Yes & yes & no & 36.5 \\
Yes & no & yes & 44.1 \\
No & no & no & 53.6 \\
Yes & no & no & 63.2 \\
\hline
\end{tabular}

0-MM is zero mismatch or a 6-antigen match kidney transplant. FSGS $=$ focal segmental glomerulosclerosis, $L D=$ living donor .

Table 3: Cox proportional hazard model covariates for death-censored graft survival

\begin{tabular}{lllc}
\hline & RR & $95 \% \mathrm{Cl}$ & $\mathrm{p}$-value \\
\hline Recipient age & 0.999 & $0.997-1.002$ & 0.667 \\
Recipient gender & 1.002 & $0.940-1.067$ & 0.956 \\
African American & 1.870 & $1.745-2.005$ & $<0.0001$ \\
MMF & 0.793 & $0.664-0.948$ & 0.011 \\
FK506 & 1.020 & $0.849-1.226$ & 0.831 \\
Induction & 1.069 & $0.769-1.488$ & 0.691 \\
CIT (h) & 1.004 & $1.001-1.007$ & 0.019 \\
PRA (\%) & 1.004 & $1.003-1.006$ & $<0.0001$ \\
Donor age & 1.014 & $1.012-1.016$ & $<0.0001$ \\
\hline
\end{tabular}

Adjusted for time on dialysis; reference group for African American race is Caucasian cohort; reference group for MMF is azathioprine; reference group for FK506 is cyclosporine.

PRA $=$ panel reactive antibody, $\mathrm{CIN}=$ cold ischemic time,

$\mathrm{MMF}=$ mycophenolate mofetil.

Annually adjusted graft loss rates calculated from the Cox model are displayed in Table2. For FSGS patients with a zero mismatch LD renal transplant, the annually adjusted graft loss rate was 10.5 per 1000 patient-years as compared to the GN reference group whose annually adjusted graft loss rate was 14.3 per 1000 patient-years. In comparison, those FSGS patients who received a mismatched LD renal transplant had an annually adjusted graft loss rate of 36.5 per 1000 patientyears. In contrast, the FSGS patients with a zero mismatch CAD renal transplant or with a mismatched CAD renal transplant had annually adjusted graft loss rates of 44.1 per 1000 patient-years and 63.2 per 1000 patient-years, respectively.

Covariates for the Cox proportional hazard model for deathcensored graft survival are shown in Table 3. Of the 11 covariates in the model, donor age, PRA, CIT, African American race, and time on dialysis (data not shown) were significant risk factors for death-censored graft failure. In contrast, mycophenolate mofetil was a significant protective factor against death-censored graft failure. Recipient age and gender, induction, and FK506 were not significant factors for death-censored graft survival.

\section{Discussion}

Currently in some centers, zero mismatch LD kidney transplantation has been avoided in FSGS patients for fear that 
recurrent FSGS in the renal allograft would lead to premature graft loss, even though graft survival among LRD transplant recipients is superior in well-matched grafts and is far superior to the graft survival rate among CAD transplant recipients. In contrast to this widespread concept, our study demonstrates superior death-censored graft survival for FSGS patients who received a zero mismatch LD kidney transplant as compared to those FSGS patients who received an HLAmismatched cadaveric kidney transplant. Furthermore, the FSGS patients who received a zero mismatch LD kidney transplant had similar death-censored graft survival as compared to the GN patients with a zero mismatch LD kidney transplant. Glomerulonephritis patients were used as the reference population because these patients reflect a homogeneous population of patients without pre-existing comorbidities, similar to those patients with FSGS. In addition, among patients with ESRD, GN patients have been demonstrated to have superior death-censored graft survival $(15,16)$, so we compared our FSGS cohort to a reference population known to have excellent renal allograft survival.

As mentioned earlier, previous studies and current literature on renal transplantation suggest that LRD transplants for patients with FSGS give a high rate of recurrence and hence, portend a worse outcome as opposed to cadaveric renal transplants. As recurrence of FSGS has been related to worse graft survival, these studies suggest that a zero mismatch LRD kidney transplant should be avoided in patients with ESRD from FSGS. In fact, this recommendation is widely held by U.S. transplant centers today. Although our data does not address the incidence of recurrent FSGS in patients with a LRD kidney transplant, it does address renal allograft survival in FSGS patients who received a LD kidney transplant as compared to those FSGS patients who received a cadaveric kidney transplant. In addition, we address the issue of graft survival in those FSGS patients who received a zero mismatch LD kidney transplant. Censoring for death, our data shows that FSGS patients who had a zero mismatch LD kidney transplant had significantly superior graft survival over FSGS patients who received either a zero mismatch or HLAmismatch CAD kidney transplant. Therefore, ESRD patients with FSGS benefit from live donation and a zero mismatch, and, if possible, should receive a zero mismatch LD kidney transplant to maximize chances for long-term graft survival.

As with any retrospective analysis, there are limitations to the study. It cannot be determined if the diagnosis of FSGS was biopsy-proven, even though it is unlikely to assume that any nephrologist would diagnose FSGS as the primary cause of ESRD without a biopsy. In addition, some secondary causes of FSGS may have been recorded as the primary cause of ESRD. Causes of graft loss and incidence of recurrence have been reported previously and hence were not the focus of our analysis $(12,13)$.

In summary, zero mismatch LRD kidney transplants are not a risk factor for graft loss in FSGS patients but are associated with significantly better death-censored renal allograft sur -vival as compared to cadaveric zero mismatch or HLA-mismatch donations.

\section{Acknowledgements}

The data reported here have been supplied by the U.S. Renal Data System (USRDS) and the U.S. Scientific Renal Transplant Registry. The interpretation and reporting of these data are the responsibility of the authors and in no way represent an official policy or interpretation of the U.S. Government.

\section{References}

1. Orth SR, Ritz E. The nephrotic syndrome. N Engl J Med 1998; 338: 1202-1211.

2. Savin VJ, Sharma R, Sharma M et al. Circulating factor associated with increased glomerular permeability to albumin in recurrent focal segmental glomerulosclerosis. N Engl J Med 1996; 334: 878-883.

3. Artero M, Biava C, Amend W, Tomlanovich S, Vincenti F. Recurrent focal glomerulosclerosis: natural history and response to therapy. Am J Med 1992; 92: 375-383.

4. Banfi G, Colturi C, Montagnino G, Ponticelli C. The recurrence of focal segmental glomerulosclerosis in kidney transplant patients treated with cyclosporine. Transplantation 1990; 50: 594-596.

5. Hariharan S, Adams MB, Brennan DC et al. Recurrent and de novo glomerular disease after renal transplantation: a report from Renal Allograft Disease Registry (RADR). Transplantation 1999; 68: 635641.

6. Feld SM, Figueroa P, Savin $\mathrm{V}$ et al. Plasmapheresis in the treatment of steroid-resistant focal segmental glomerulosclerosis in native kidneys. Am J Kidney Dis 1998; 32: 230-237.

7. First MR. Living-related donor transplants should be performed with caution in patients with focal segmental glomerulosclerosis. Pediatr Nephrol 1995; 9 (Suppl.): S40-S42.

8. Zimmerman CE. Renal transplantation for focal segmental glomeruIosclerosis. Transplantation 1980; 29: 172.

9. Morzycka M, Croker BP Jr, Siegler HF, Tisher CC. Evaluation of recurrent glomerulonephritis in kidney allografts. Am J Med 1982; 72: 588-598.

10. Al-Akash SI, Ettenger RB. Kidney transplantation in children. In: Danovitch GM, ed. Handbook of Kidney Transplantation. Philadelphia: Lippincott Williams \& Wilkins, 2001: 340

11. Sayegh MH, Kaplan AA. Focal Glomerulosclerosis: Recurrence After Transplantation. Rose BD, ed. Up-to-date [Version 8.3]. UpToDate Inc., 2000.

12. Baum MA, Stablein DM, Panzarino VM et al. Loss of living donor renal allograft survival advantage in children with focal segmental glomerulosclerosis. Kidney Int 2001; 59: 328-333.

13. Abbott KC, Sawyers ES, Oliver JD et al. Graft loss due to recurrent focal segmental glomerulosclerosis in renal transplant recipients in the United States. Am J Kidney Dis 2001; 37: 366-373..

14. Cecka JM. The UNOS Scientific Renal Transplant Registry. In: Cecka JM, Terasaki PI. Clinical Transplants. Los Angeles: UCLA Immunogenetics Center, 1999: 1-21.

15. Meier-Kriesche HU, Ojo AO, Cibrik DM et al. Relationship of recipient age and development of chronic allograft failure. Transplantation 2000; 70: 306-310.

16. Meier-Kriesche HU, Ojo AO, Leichtman AB et al. Interaction of mycophenolate mofetil and HLA matching on renal allograft survival. Transplantation 2001; 71: 398-401. 